\title{
LEPROSY: THE TASKS FACING SCIENTIFIC RESEARCH
}

by Candidate of Medical Sciences V. K. LoginOV

of the Scientific Research Institute for the Study of Leprosy

(Director: Candidate of Medical Sciences V. F. SHuBIN)

(Reprinted from the Journal of Dermatology and Venerology', No. 5, 1963, Moscow, pp. 41-45, in English translation from the original Russian text hy the kindness of Mr. D. M. Blakeley in collahoration with Dr. J. Ross Innes.)

During the past two decades our knowledge of leprosy has made considerable advances. There have been definite achievements in diagnosis, treatment and prevention. Leprosy may now be diagnosed in the very earliest stage of its development and, in certain cases, even before the appearance of visible symptoms. All this has been achieved as a result of a whole complex of clinical, functional and pathohistological research.

In cases of malignant (lepromatous) leprosy present-day forms of treatment are able comparatively quickly to stop the spread of the leprosy bacillary focus into the surrounding area by achieving socalled bacterial negativity. Early diagnosis and effective treatment have made it possible, on a comparatively large scale, to transfer leprosy patients to outpatient treatment. All this has undoubtedly helped to alter the age-old view of leprosy as a highly infectious incurable disease and to overcome vulgar panic-stricken fear of the leprosy sufferer.

As a result, certain legislation regarding leprosy has been changed in many countries, including the Soviet Union; in particular the regulations relating to the hospitalisation of patients have been reviewed and dispensary methods more and more widely adopted in leprosy treatment.

However, these achievements were based for the most part on empirical data. There still remains a great deal that is unclear and unsure in our knowledge of the disease.

The great problem is to discover ways of obtaining the cultures of the leprosy bacterial focus in human beings. In spite of the great body of research already carried out, no research worker has yct been able to discover a method of obtaining cultures from a leprosy patient. It is true that there have recently been indications of the possibility of cultivating mycobacteria in tissue cultures, but these require verification.

Our knowledge of the biochemistry of the leprosy bacteria in man is very meagre indeed.

In regard to the diffusion of Mycobacterium leprae in man in its viable form, it would seem a possibility in the future, using bio- 
chemical methods, to obtain more precise data about its chemical composition, and its fermentative and other properties. Of particular interest, especially for diagnosis and prophylaxis, is the study of the antigenic properties of albuminous and other fractions obtained both from the leprosy bacilli and from infected tissues.

Undoubtedly, the greatest hope for progress lies in a study of the morphology of the leprosy bacilli by means of the electron microscope. From our point of view this research should be carried out during the course of treatment using tissue culture and growth in symbiosis.

Research into experimental leprosy is particularly important. Here, quite obviously, an investigation of the forms of leprosy in human beings is fundamental. It is difficult to say beforehand which particular channel of investigation will be found to be most profitable but it goes without saying that those who undertake research in this ficld should make a very careful study and analysis of the mistakes of their predecessors.

Another promising field of possible development is the comparative study of the immunological, pathological, morphological and biochemical properties of the leprosy bacillus in man and in mice, and of other mycobacterial diseases in animals in order to determine more precisely the connection between the respective forms. There is definite practical and theoretical significance in the study of the mutual connection-clinical, epidemiological and immunologicalof leprosy and tuberculosis, and also of certain paratuberculous diseases. The leprosy-like diseases in mammals, birds, fish, reptiles, etc., have not been widely studied.

Of particular interest is the study of leprosy in mice (Stefanov's disease) which, because of its many parallel symptoms, can serve, at least in part, as an accepted model for leprosy in human beings. However, it must be remembered that the bacilli of this disease are nearer to the tuberculous and paratuberculous bacilli than to the leprosy bacilli in man (Chaussinand, N. A. TORSUYEv).

One of the fundamental criteria in judging the resistance of the body to leprosy infection is the lepromin reaction. It has been proved that healthy persons with a positive lepromin reaction very rarely succumb to leprosy, and if the disease does develop it usually takes a benign course. On the other hand, people with a negative lepromin reaction have less resistance to leprosy; with them the disease is usually malignant. From this it follows that the strengthening of the lepromin reaction in healthy people and in leprosy patients is an indicator of the resistance of the body to leprosy infection.

In this connection there is definite theoretical and practical importance in attempts artificially to strengthen the lepromin reaction in healthy people who have been in contact with leprosy patients. In particular, the search for ways and means of strengthen- 
ing the lepromin reaction is of some interest. At the same time convincing proof must be forthcoming that this is accompanicd by an increase in the resistance of the body to leprosy infection.

Specific vaccines, which might boost the defensive resources of the organism and, in leprosy patients, prevent the spread of the disease, are yet to be found. The solution to this problem of increasing the resistance of the human body to leprosy infection must therefore be approached by indirect methods, using other unspecific substances.

Interest has increased in the study of the defensive effect of BCG vaccination in leprosy contacts and patients. An established effect of this vaccine in leprosy patients is to stimulate a change-over from a negative to a positive lepromin reaction. There is much evidence to show that the morbidity of leprosy among people vaccinated with BCG is less than among those not so vaccinated. However, the defensive role of the vaccine in the prophylaxis of leprosy is not definitely proven. A study should be made of the possible methods of using this vaccine (cutaneous, intracutaneous, peroral, etc.). A study of the BCG vaccine in the prophylaxis of leprosy should most usefully be undertaken, on a mass scale, in those areas where leprosy is most endemic. These measures should be carried out jointly with measures against tuberculosis, given the simultaneous prophylaxis of both leprosy and tuberculosis.

Studies must also be made of other antigens which are capable of increasing resistance to leprosy. Repeated doses of the Mitsuda lepromin itself, though it has only a weak antigenic power, may also strengthen lepromin reaction. However, in this case it has not been established whether any changes are caused in the body which encourage an increase in the resistance to leprosy. In this connection a study should be made of other antigens and stimulants (Stefanskiy's lepromin, Kedrov's antigen, Friend's stimulant, etc.).

The chemoprophylaxis of leprosy has not been widely studied. Several authors have reported that amongst children in contact with the disease, treated with subtherapeutic doses of DDS, the morbidity of leprosy is considerably less than amongst children who were not treated. During breast-feeding a child receives a medicinal preparation in sufficient quantity from the milk of his mother, who is taking sulphones. Some authors recommend that after weaning, or after birth if the mother is not receiving treatment, the child should be put on a course of sulphone treatment consisting of $5 \mathrm{mg}$. a week for each $1 \mathrm{~kg}$. of weight. However, as regards the problem of whether it is profitable to use similar chemoprophylaxis in the case of leprosy, there is still a great deal that is controversial and unsubstantiated.

At the present time it has become rather difficult to get the antigen of lepromin because of the absence of initial material for its preparation namely the tissues of lepromatous leprosy patients containing 
Mycohacterium leprae. Due to earlier diagnosis and adequate treatment active tissue material is less often obtainable. Moreover, the demand for lepromin grows from year to year. Therefore the search for new methods of obtaining it-and the use of other substances in its place-becomes of prime importance. In order to economise on lepromin it can be used in larger dilutions than is normally accepted.

The absence of a tissue-free leprosy antigen is a spur to research into the formation of a specific antigen free from tissue material.

The extraction of the most active fractions of lepromin, or of Mycohacterium leprae, would provide an answer to the problem concerning the nature of both the late and the early lepromin reaction.

The use of serological reactions in leprosy runs into difficulty because of the absence of a leprosy antigen. However, experience shows that these reactions may be studied with antigens prepared from other germs as of tuberculosis, syphilis, etc.

On examination of the blood serum of lepromatous leprosy patients with paraspecific antigens, considerably more antibodies may be found than is the case with healthy people. Moreover, the skin reaction to lepromin in these patients is almost always negative, indicating a state of anergia. This contradiction calls for a simultaneous study of serological reactions and skin reactivity in leprosy. Parallel research into the reactivity of mycobacterial antigens of differing origin, and a comparison of these reactions in clinical and experimental situations, might help in the study of many problems connected with the immunology of leprosy.

There is a pressing need for a study in depth of the epidemiology of leprosy in general and, in particular, of each focus (determining the boundaries of each focus, the rate of infection and the dynamics of development, the course and the dying away of the focus, dissemination, the formation of new foci, etc.). This would facilitate the planning of new and better methods of attack in the battle against leprosy.

As with other diseases, one of the factors governing the spread of leprosy is its infectivity. However, other very important factors should be taken into account. One of these is individual human susceptibility. For example, by no means everyone who has had close and prolonged contact with lepromatous leprosy patients acquires the disease. This explains the low incidence of leprosy amongst the husbands or wives of patients and the rare cases of infection amongst leprosaria staff.

Many attempts have been made to infect people artificially with leprosy. But they have all failed. For example over a period of 14 months Guy Prieto and Contreras transfused into a 26 year old subject the blood of leprosy patients in an exacerbated state. They also administered to their subject nasal mucus from lepromatous 
patients containing a large quantity of $M$. leprcie and grafted on to him pieces of biopsied tissue from a leprosy patient. During subsequent investigations over an 8-year period no traces of leprosy were to be found in the subject. Although we consider similar experiments on human beings to be inadmissible, we cannot fail, nevertheless, to take their results into account.

Obviously, we cannot countenance the obligatory isolation of all leprosy patients, since it is frequently a hindrance to measures being carried out to combat the disease. According to conclusions reached by the Pan-American Conference (1958) and the African Conference at Brazzaville (1959) the compulsory isolation of patients in leprosaria is a positive obstacle to control of the disease, since many sufferers, fearing isolation, hide themselves away.

On the question of separating healthy children from parents suffering from leprosy there is much controversy. Experience in some countries has indicated that to put such children in special preventive institutions has made their return to society difficult and has sometimes been the cause of psychological trauma with serious consequences.

Because of the absence of experimental models of leprosy in man, the study of the effectiveness of medical preparations is still carried out, without intermediary, on the patient himself. In these circumstances bursts of unjustified enthusiasm, followed by gradual disappointment, are inevitable. One example of this in recent years was the persistent recommendation by certain authoritative leprologists of isoniazides and thiosemicarbasones as effective treatments for leprosy; these have failed to prove themselves during more prolonged investigation.

The discovery of sulphone preparations in 1908 and the subsequent confirmation of their effectiveness (FAGET and colleagues) was a revolutionary development for leprology. For leprosy treatment at present there are basically two sulphone preparations which are used : DDS (diaminodiphenylsulphone) and Sulphetrone (a soluble sulphone). Comparatively recently there were reports of the effectiveness of CIBA 1906 (DPT) which is derived from thiourea. In the Soviet Union clinical tests have been carried out on Etoxid, which is also derived from thiourea. The results have shown that it is effective in the treatment of leprosy. Undoubtedly worthy of close attention are the many reports testifying to the positive effectiveness of Etisul (a derivative of ethylmercaptan). This preparation, which in itself has little influence on the course of the disease, increases to a quite significant extent the therapeutic effectivity of sulphones, particularly of DDS. Of the quite numerous new substances recommended for the treatment of leprosy, mention should be made of Vadrine (oxidiazalone) and Cycloserine. Soviet leprologists have no experience of the use of these last two preparations. 
As for the future of leprosy treatment, there are two directions for development. One of these is to improve existing methods of leprosy treatment by determining the optimum doses, confirmed by experiment, for treatments; and by calculating precisely their distribution in the organism and the times at which the preparation is administered. Given the above, it may be possible in the future to use drug combinations and to study the feasibility of using several treatments simultaneously; also to work out the most effective rotations of the above and a combination of anti-leprosy and other unspecific effective methods which prevent the development of toxic manifestations and of other side effects. At the same time the search must go on for new highly-effective anti-leprosy preparations with minimal toxic properties. The appropriate methodology must be used for the evaluation of new preparations for leprosy treatment. Observations should be carried out only on lepromatous-type patients, and as far as possible on those who have not previously been treated. A group of patients being treated with DDS in the usual dosages must be included in these observations.

Also of very great importance is the elimination of cosmetic defects and orthopaedic lesions due to leprosy or secondary to it. At the Scientific Research Institute for the Study of Leprosy, nasal deformity is already being corrected with the aid of a specially prepared prosthesis made from plastic and also free skin plastic surgery of the nose and of the eyebrow with favourable cosmetic and functional results. In the press recently there were reports of muscular atrophy in leprosy being treated successfully by local injections of Vitamin E.

There is still a good deal of obscurity in the theory and practice of leprology. For the thoughtful research worker there is still much to do. The majority of the unanswered questions in leprology are closely bound up with the present-day problems of modern medicine and biology.

"It should never be forgotten", says the famous leprologist R. G. Cochrane, "that leprosy is not a shameful social disease, but a deeply interesting problem which deserves the attention of the best scientific minds and opens up a wide field for research".

\section{Summary (added by the Editor)}

The author writes from out of his experience and that of his colleagues at the Institute for the Study of Leprosy at Astrakhan and makes valuable suggestions for research and the modern control of leprosy. He emphasises that the chief priority is to stop the spread of the leprosy bacillus, both in the body and in the geographical area, and that modern drug treatment has achieved a great deal in this regard. It is, however, urgent to continue research for quicker and more effective drugs and he mentions some of them which have 
been tested, particularly Etoxid which so far has achieved excellent therapeutic results in Russia. See Leprosy Review, 34, 4. Oct. 1963, pp. 212-218.

The greatest problem is how to culture the leprosy bacillus and though tissue culture has been achieved it has not obtained practical application. Another urgent need is the discovery of an effective vaccine, whether based on human leprosy or on related diseases in man or animals. In the meantime, he thinks that BCG is not to be despised and mentions evidence that the morbidity of leprosy among people vaccinated with $B C G$ is less than among those not vaccinated. $\mathrm{He}$ mentions that repeated doses of the Mitsuda lepromin itself may also strengthen the positive reaction to leprosy, and calls for a study of other antigens. He calls for a simultaneous study of serological reactions and skin reactivity in leprosy. He points out that there is a pressing need for study in depth of the epidemiology of leprosy in general and of each focus in particular.

On using modified dosage of anti-leprosy drugs to prevent the disease in children, he things the evidence is equivocal.

For deformities in leprosy, plastic and reconstructive surgery have found a very important and satisfactory place.

The author's paper well repays study in the complete form.

J. R. INNES, Editor.

\section{References}

TorsuYev, N. A. BME. M., vol. 26,1962 , p. 867.

Chaussinand, R., Bull. Acad. Nat. Med. (Paris), 1948, vol. 182, p. 486.

Cochrane, R. G. Leprosy in Theory and Practice. Bristol, 1959.

Faget, G. H. et al., Publ. Hlth. Rep. (Wash.), 1943, vol. 58, p. 1729.

Prieto, J. G., Contreras, F. B. Memoria del 6 Congreso Internat. de Leprologia, Madrid, 1953, p. 475. 